\title{
Effet de l'inversion du cycle jour-nuit sur le moment du poulinage au cours du nycthémère chez les chevaux domestiques
}

\author{
M.J. BOSC, G. DUCHAMP et E. RODAS ${ }^{(1)}$ \\ INRA, Station de Physiologie de la Reproduction, \\ Centre de Recherches de Tours, Nouzilly, F 37380 Monnaie
}

\begin{abstract}
Résumé
Les moments de poulinage ont été comparés chez deux lots de juments de race Welsh et Selle français. Le lot témoin (lot T) a été exposé à l'éclairement naturel tandis que le lot inversé (lot I) a été soumis à un cycle «jour-nuit » inversé. Ces conditions lumineuses ont débuté environ 40 jours avant les premières mise bas. Les autres conditions d'élevage ont été maintenues aussi semblables que possible dans les deux lots. Dans le lot T, 12 animaux sur 15 (80 p. 100), dans le lot $I, 7$ juments sur $12(58,3$ p. 100) ( $p>0,05)$ ont mis bas durant leurs nuits respectives. Ces résultats indiquent que la photopériode exerce un effet déterminant sur le système endogène dont dépend le moment de la naissance chez les chevaux domestiques. Ils suggèrent que le moment de la naissance dans cette espèce peut être contrôlé par des conditions d'environnement appropriées.
\end{abstract}

Mots clés : Cheval, mise bas, moment, cycle nycthéméral, inversion.

\section{Introduction}

Parmi les animaux domestiques, la jument est bien connue pour pouliner pendant la nuit (Zwolinski, 1965 ; Rossdale \& Short, 1967 ; JeffCoat, 1972 ; Campitelli et al., 1982-1983 ; TAINTURIER et al., 1984). Cet événement peut dépendre directement de la photopériode, il peut aussi être la conséquence de facteurs environnementaux secondaires qui sont eux-mêmes contrôlés par la photopériode. A cet égard, les conditions d'élevage ont été considérées comme importantes (ZwolıNSKI, 1965 ; RossDALE \& ShORT, 1967 ; Campitelli et al., 1982-1983). Afin d'éprouver si la photopériode contrôle par elle-même le moment du poulinage, nous avons comparé deux groupes de juments maintenues à l'intérieur et soumises à l'éclairement naturel ou à un cycle « jour-nuit » inversé.

(1) Adresse actuelle : Instituto de Ciencias Fisiologicas, Facultad de Veterinaria, Alberto Lasplaces 1620, Montevideo, Uruguay. 


\section{Matériel et méthodes}

Dix-neuf juments de race Welsh et 8 juments de Selle français ont été utilisées. Elles ont été inséminées après un oestrus induit (Driancourt \& Palmer, 1982), un ou deux jours avant l'ovulation qui a été déterminée par un examen échographique de routine (Palmer \& Driancourt, 1980). Le jour de l'ovulation a été considéré comme étant le jour 0 de la gestation. Compte tenu des dates d'ovulation, les poulinages ont été attendus au cours des mois de juin et de juillet. Deux lots ont été formés de façon à ce qu'ils soient équilibrés pour les dates d'insémination. Ils ont été placés dans deux pièces contiguës d'un grand bâtiment environ 40 jours avant les premiers poulinages (soit fin avril). Le lot témoin (lot $\mathrm{T}$ ) a été exposé à l'éclairage naturel. Selon les positions relatives des ouvertures et des boxes, la luminosité a été très variable ; ainsi avec un ciel nuageux, la luminosité a été comprise entre 2 et 120 lux (Luxmètre CL 2010, Chauvin et Arnoux, Paris) à mi-journée aux niveaux de la tête des animaux. L'autre lot (lot I) a été soumis à un cycle "jour-nuit » inversé. Cette inversion a été réalisée progressivement en six jours et elle a été accompagnée par une légère réduction de la durée de la phase obscure par rapport à celle que subissaient les témoins à la même époque. Dans ce lot (lot I) la lumière a été donnée entre $16 \mathrm{~h}$ et $8 \mathrm{~h} 30$ de façon que cette durée d'éclairement soit approximativement égale à celle du lot $\mathrm{T}$ au moment du solstice d'été sous notre latitude $\left(47,5^{\circ}\right.$ nord). La pièce accueillant le lot $I$ avait été préalablement isolée de toute source lumineuse extérieure. L'éclairage de cette pièce, entièrement dispensé par des ampoules ordinaires, a assuré une luminosité comprise entre 10 et 40 lux aux niveaux des juments.

Les autres conditions d'élevage ont été maintenues aussi semblables que possible dans les deux lots. Les repas ont été distribués individuellement lors des périodes claires communes aux deux lots, entre $7 \mathrm{~h}$ et $8 \mathrm{~h} 30$ le matin et entre $16 \mathrm{~h}$ et $17 \mathrm{~h}$ au cours de l'après-midi. Les juments ont pris un exercice hebdomadaire à l'extérieur entre $7 \mathrm{~h}$ et $8 \mathrm{~h} 30$ lors du curage de leur box. A tout autre moment de la journée, les deux pièces ont été fermées. Les températures maxima et minima ont été enregistrées chaque jour ; elles ont varié de la même façon dans les deux pièces (coefficient de corrélation égal à 0,94$)$ et leurs moyennes se sont établies respectivement à $22,6^{\circ} \mathrm{C}(\mathrm{sd}= \pm 3,1)$ et $21,9{ }^{\circ} \mathrm{C}(\mathrm{sd}= \pm 2,2)(\mathrm{P}>0,05)$ dans la pièce du lot $\mathrm{T}$ et celle du lot $\mathrm{I}$. Enfin le jour ou le lendemain du poulinage, la jument et son poulain ont été placés au pré.

Les surveillances ont été faites trois fois par jour : aux moments des repas et vers $22 \mathrm{~h} 30$. Les repas délimitent le jour et la nuit dans le lot $\mathrm{I}$; le repas de $7 \mathrm{~h}$ et la surveillance effectuée à $22 \mathrm{~h} 30$ correspondent aux moments équivalents dans le lot $\mathrm{T}$. Les taux de poulinage ont été déterminés pour chacune des trois périodes quotidiennes ainsi délimitées ; ils ont été comparés entre eux selon le test de $\mathrm{X}^{2}$ (VessereaU, 1960).

\section{Résultats}

Les taux des mise bas observées pendant les trois périodes de la journée définies par les conditions photopériodiques sont présentées dans le tableau $\mathrm{n}^{\mathbf{0}} 1$; ils diffèrent entre les deux groupes $(\mathrm{p}<0,01)$. Cependant dans chaque groupe, les taux de mise bas 
TABLEAU 1

Nombre (n) et taux (\%) des poulinages chez les lots témoin (lot T) ou inversé (lot I) selon les conditions d'éclairement et les périodes de la journée.

Number ( $n$ ) and rates (\%) of foaling in the control (T) and inverted (I) groups according to the lighting conditions and the periods of the day.

\begin{tabular}{|c|c|c|c|c|c|c|c|c|c|}
\hline \multirow{4}{*}{ Lots / Groups } & \multicolumn{9}{|c|}{ Périodes de la journcé / Periods of the day } \\
\hline & \multicolumn{3}{|c|}{8 h $30-16$ h 30} & \multicolumn{3}{|c|}{16 h $30-22$ h 30} & \multicolumn{3}{|c|}{22 h $30-8$ h 30} \\
\hline & \multirow{2}{*}{ LC } & \multicolumn{2}{|c|}{$\mathrm{MB} / F$} & \multirow{2}{*}{ LC } & \multicolumn{2}{|c|}{$\mathrm{MB} / F$} & \multirow{2}{*}{$\mathbf{L C}$} & \multicolumn{2}{|c|}{$\mathrm{MB} / F$} \\
\hline & & $\mathbf{n}$ & $\%$ & & n & $\%$ & & $\mathbf{n}$ & $\%$ \\
\hline$\ldots \ldots \ldots$ & L & 2 & 13,3 & $\mathbf{L}$ & 1 & 6,7 & D & 12 & 80,0 \\
\hline$I \ldots \ldots \ldots \ldots$ & D & 7 & 58,3 & L & 3 & 25,8 & $\mathbf{L}$ & 2 & 16,7 \\
\hline
\end{tabular}

LC : Conditions d'éclairement /Lighting conditions ; L : Lumière /Light ; D : Obscurité / Darkness.

MB : Mise bas ; $F$ : Foaling.

qui se sont produites pendant les phases obscures respectives, ont été équivalents ( $p>0,05)$; ils représentent 80 p. 100 des juments du lot T et 58,3 p. 100 des juments du lot $\mathrm{I}$. Les poulinages 's'étant déroulés sur deux mois, il faut noter une variation non négligeable $(p>0,05)$ du nombre des mise bas de nuit dans le lot $I: 4 / 5$ (80 p. 100) des poulinages se sont passés la nuit au cours du premier au lieu de $3 / 7$ (43 p. 100) au cours du second, ce qui n'a pas été observé pour le lot $\mathbf{T}$.

Les durées de gestation ont été indépendantes des conditions photopériodiques

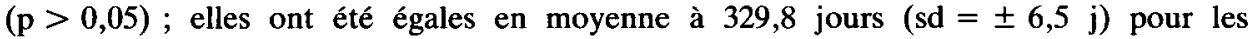
juments Welsh et à 328,6 jours (sd $= \pm 9,6 \mathrm{j}$ ) pour les juments de Selle. Compte-tenu des répartitions des juments par lot, les durées passées dans chaque lot ont été équivalentes $(p>0,05)$ (en moyenne 65,9 jours) bien que fort variables (extrêmes : 40 et 96 jours).

Dans le lot I, deux poulains de Selle ont été trouvés morts après une naissance de nuit ; les raisons de leur décès n'ont pas pu être établies. Il n'y eut pas d'autres pertes à déplorer à la naissance comme au cours des deux premiers mois de lactation.

\section{Discussion}

Cette expérience démontre que la photopériode exerce un effet déterminant sur le système endogène dont dépend le moment de la naissance chez les chevaux domestiques. L'inversion du cycle « jour-nuit » a entraîné le déplacement d'une forte proportion de mise bas et dans le lot inversé comme dans le lot témoin, la majorité des mise bas se sont produites de nuit. Chez les témoins, la distribution nycthémérale des mise bas est identique à celles qui ont été auparavant rapportées (ZwolINSKI, 1965 ; Rossdale \& Short, 1967 ; JefFCOAT, 1972 ; CAMPITElli et al., 1982-1983 ; TAINTURIER et 
al., 1984). Dans notre expérience, cette distribution a été observée dans des conditions strictes d'obscurité alors que dans d'autres études (JEFFCOAT, 1972), un éclairage d'appoint a pu être dispensé au cours des dernières nuits de la gestation. La distribution nycthémérale des poulinages est donc le résultat de rythmes endogènes établis avant la parturition en fonction de l'alternance du jour et de la nuit.

Dans le lot ayant subi l'inversion du cycle « jour-nuit», une diminution sensible du nombre des poulinages de nuit a été relevée entre le premier et le deuxième mois au cours desquels se sont déroulées les mise bas. Il est probable que des conditions d'environnement autres que la photopériode, aient modulé la réponse photopériodique, car à ce sujet, il faut noter que les abords du bâtiment expérimental ont été plus bruyants pendant les jours ouvrés du second mois par comparaison à ceux du premier. L'effet de la photopériode pourrait donc être amoindri ou renforcé par d'autres facteurs environnementaux qui restent à préciser.

Les effets secondaires liés aux conditions expérimentales ont été limités à l'observation de la survie des poulains. Deux poulains de Selle sont morts et il est difficile de rendre le traitement lumineux responsable de ces pertes. Lorsque tous les animaux sont regroupés, le taux de la mortalité périnatale est égal à 7,4 p. 100 ce qui correspond aux taux observés dans notre pays (TAINTURIER et al., 1984).

La photopériode qui paraît être la cause déterminante pour laquelle la jument pouline préférentiellement de nuit met en jeu des mécanismes physiologiques encore inconnus. Quoiqu'il en soit, ces résultats offrent des perspectives intéressantes, en particulier pour le contrôle du moment de la naissance. On sait que la parturition peut être induite par diverses drogues chez cette espèce (JefFCoAt \& Rossdale, 1977) mais la prématurité qui en résulte affecte la viabilité des jeunes (LEAdon et al., 1986). Pour éviter ces problèmes et pour rendre les surveillances plus aisées, le déplacement du moment de la naissance aux heures ouvrées de la journée est une alternative séduisante. Nos résultats montrent que l'inversion du cycle «jour-nuit » peut être une solution sans augmentation apparente des effets secondaires indésirables. Il reste alors à déterminer le temps minimum requis pour que cette inversion soit efficace. Ces résultats suggèrent aussi que tout moyen qui permet de mimer ou de modifier un cycle « jour-nuit » chez la jument domestique, peut être adapté au contrôle du moment de la naissance.

Reçu en juin 1988.

Accepté en septembre 1988.

\section{Remerciements}

Cette étude a pu être réalisée grâce aux soutiens de l'Institut National de la Recherche Agronomique et du Service National des Haras (Ministère de l'Agriculture, France ; Contrat $\mathrm{n}^{\circ}$ 86006). Nous remercions Mademoiselle M.E. ChOISNET pour sa participation active. 


\author{
Summary \\ Effect of inversion of the light-dark cycle on foaling time during \\ the day in domestic horses
}

Foaling times were compared in two groups of mares of Welsh and Selle Français breeds. They were maintained into two contiguous rooms. The control group (group T) was kept under the natural lighting conditions. The second group (group I) was exposed to an inverted light-dark cycle. The light treatment started about 40 days before the first expected births. The other husbandry conditions were maintained as similar as possible in both groups. In the group $\mathrm{T}$, 12 animals of $15(80 \mathrm{p}$. 100) foaled between $22: 30$ and $8: 30 \mathrm{~h}$ during the night. In the group I, 7 mares of $12(58.3 p .100)(p>0.05)$ gave birth between 8:30 and 16:30 $h$ during their own dark phase. These results demonstrate that photoperiod exerts a determinate effect on the endogenous system which controls foaling time in domestic horses. They suggest that birth time in this species may be controlled by appropriate environmental conditions.

Key words : Horse, foaling time, light-dark cycle, inversion.

\title{
Références bibliographiques
}

Campitelli S., Carenzi C., Verga M., 1982-1983. Factors which influence parturition in the mare and development of the foal. Applied Animal Ethology, 9, 7-14.

Driancourt M.A., Palmer E., 1982. Seasonal and individual effects on ovarian and endocrine responses of mares to a synchronization treatment with progestagen-impregnated vaginal sponges. J. Reprod. Fert., Suppl., 32, 283-291.

JefFCOAT L.B., 1972. Observations on parturition in crossbred pony mares. Equine Vet. J., 4, 209213.

Jeffcoat L.B., Rossdale P.D., 1977. A critical review of current methods for induction of parturition in the mare. Equine Vet. J., 9, 208-215.

Leadon D.L., Jefrcoat L.B., Rossdale P.D., 1986. Behavior and viability of the premature neonatal foal after induced parturition. Am. J. Vet. Res., 47, 1870-1873.

Palmer E., Driancourt M.A., 1980. Use of ultrasonic echography in equine gynecology. Theriogenology, 13, 203-216.

Rossdale P.D., Short R.V., 1967. The time of foaling of thoroughbred mares. J. Reprod. Fert., 13, 341-343.

Tainturier D., Chevalier F., Palmer E., Ganière J.P., Rama F., Domerg D., Marionneau G., 1984. L'élevage breton : mise à la reproduction, pertes après fécondation, état sanitaire. In "10 Journée de la Recherche Chevaline ", Ed. E. Rossier, CEREOPA, Paris, 39-48.

VesSEREAu A., 1960. Méthodes statistiques en biologie et agronomie. In "Recherches et Expérimentation en Agriculture ", p. 532. Eds F. Bouf \& A. Vessereau, J.B. Baillères et Fils, Paris.

Zwolinski J., 1965. Analyse de certains phénomènes liés à la reproduction chez le cheval (en polonais) Roczniki WSR w Poznaniu.zesz., 25, 227-232. 